\title{
STUDY ON TENDINOUS CONNECTIONS BETWEEN FLEXOR HALLUCIS LONGUS AND FLEXOR DIGITORUM LONGUS
}

\section{Manikanta Reddy ${ }^{* 1}$, V. Vallishri ${ }^{2}$, C. K. Lakshmi Devi ${ }^{3}$.}

${ }^{* 1}$ Department of Anatomy, ACSR Government Medical College, Nellore, AP, India

${ }^{2}$ Department of Pediatrics, Pondicherry Institute of Medical Sciences, Pondicherry, India

${ }^{3}$ Department of Anatomy, ACSR Government Medical College, Nellore, AP, India

\section{ABSTRACT}

Introduction: Flexor Hallucis Longus (FHL) and Flexor Digitorum Longus (FDL) are long flexors of the toes, often with the interconnecting tendinous slips at various points. These interconnecting slips hold great significance in reconstruction surgeries of ankle and foot such as chronic Achilles tendon rupture, posterior tibial tendon dysfunction (PTTD) and peroneal tendon rupture. In view of the above this study was aimed to find out various types of connections between tendons of FHL \& FDL.

Materials and methods: This cross sectional study was carried out in the Department of Anatomy ACSR Government Medical College, Nellore, conducted in a total of 34 lower limbs. Flexor digitorum brevis and abductor hallucis muscles were reflected distally after the removal of the skin, superficial fascia and plantar aponeurosis to expose FHL and FDL tendons which were examined for the interconnections. Specimens with interconnections were photographed and documented.

Result: Out of 34 samples, 17 were right sided and 17 were of left sided. Mean foot length was $22.4 \pm 1.9 \mathrm{~cm}$. Three types of connections i.e. type $1,4 \& 5$ were documented at 17 (50\%), 16 (47.1\%) and 1 (2.9\%) type 5 respectively. Type 1 tendinous connections were further classified into 3 sub types i.e. type $1 \mathrm{~A}$ were 10 (58.8\%), type 1B were $5(29.4 \%)$ and type $C$ were of 2(11.8\%).

Conclusion: This study finds maximum distribution of type1 interconnections followed by type 4 and sub type $1 \mathrm{~A}$ among type 1 . This study also reports for the first time a common origin of $1^{\text {st }}$ lumbrical from distal part of tendinous slip as well as from $1^{\text {st }}$ digital slip of FDL. These interconnections provide stable base and enhanced propulsion by toes and also act as natural tenodesis.

KEY WORDS: Flexor Hallucis Longus, Flexor Digitorum Longus, tendinous interconnecting slips.

Address for Correspondence: Dr. V. Manikanta Reddy, Assistant Professor, Department of Anatomy ACSR Government Medical College, Nellore, Andhra Pradesh, India. +91 9490907429;

E-Mail: manikantareddy.v@gmail.com

Access this Article online Quick Response code

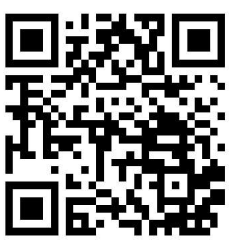

DOI: 10.16965/ijar.2019.161

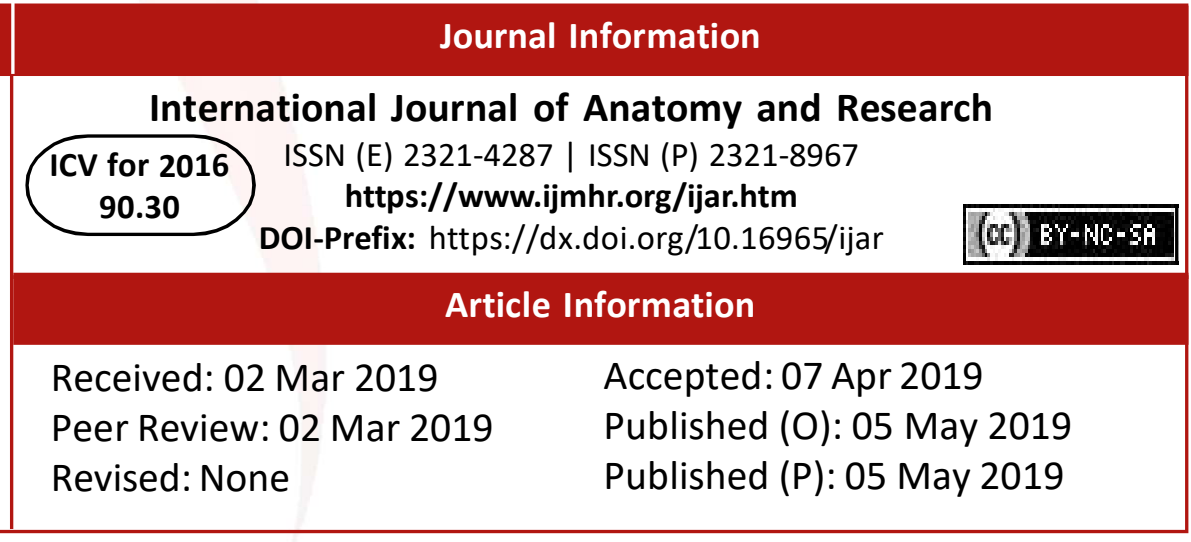

\section{INTRODUCTION}

Flexor Hallucis Longus (FHL) and Flexor Digitorum Longus (FDL) are long flexors of the toes. Tendons of these muscles present in the second layer of the sole. Tendon of the FDL crosses the tendon of the FHL from medial to lateral. This cross over is termed as 'Master knot of Henry' [1]. In the distal course of these tendons interconnecting tendinous slips are common occurrences at various points. These tendons are routinely used in reconstruction surgeries related to ankle and foot such as 
chronic Achilles tendon rupture, posterior tibial tendon dysfunction (PTTD) and peroneal tendon rupture $[2,3,4,5]$. However, there is possibility of high morbidity with vascular and neural injuries during dissection of the tendons as mobilization of tendons becomes difficult because of interconnections. These Interconnecting slip contributes to stable base, enhances the propulsion by toes while walking or running [6] and also acts as natural tenodesis [7]. Therefore, knowledge of these interconnections is crucial during the tendon graft harvesting and to understand/minimize the post-operative functional loss of toes. In view of the above this study was aimed to find out different types of connections between tendons of FHL \& FDL.

\section{MATERIALS AND METHODS}

This cross sectional observational study was carried out in the Department of Anatomy ACSR Government Medical College, Nellore. A total of 34 lower limbs from 17 formalin fixed cadavers were collected during the years 2015 to 2018. Neither deformities nor signs of surgery were found in the leg, ankle and foot regions. These specimens were subsequently dissected as follows to expose tendons of FHL and FDL. Medial and lateral plantar incisions were given to remove the skin, superficial fascia and plantar aponeurosis. This was followed by the distal reflection of flexor digitorum brevis and abductor hallucis muscles. Final exposure of FHL and FDL tendons was achieved by retracting the medial and lateral plantar arteries and nerves. FHL and FDL were examined for the tendinous interconnections between them up to their insertions. Specimens with interconnections were photographed and documented. Samples were categorised into 7 types as per the classification by Orhan Beger et al [8].

Type 1: Single slip from FHL to FDL;

Type 2: crossed connection between FHL and FDL;

Type 3: Single slip from FDL to FHL;

Type 4: no connection between FHL and FDL;

Type 5: Two slip from FHL to FDL;

Type 6: two slip from FHL to FDL and one slip from FDL to FHL;
Type 7: Two slips from FDL to FHL and one slip from FHL to FDL.

\section{RESULTS}

Total number samples analysed were 34 , among which 17 were right sided and 17 were of left sided. Mean foot length was found to be $22.4 \pm 1.9 \mathrm{~cm}$. It is interesting to note that the samples of the present study were distributed only among three types i.e. type $1,4 \& 5$. Out of 34 samples $17(50 \%)$ were of type 1 (7 right and 10 left respectively), 16(47.1\%) were of type 4 (9 right and 7 left respectively) and only $1(2.9 \%)$ was type 5(Fig: 1 ).

Type 1 i.e. single tendinous connection proximally from the FHL distally to FDL. This is further classified in to 3 sub types [8]. Out of 17 type $1,10(58.8 \%)$ were of sub type A (connection between FHL to main tendon of FDL before its division into 4 digital tendons), 5(29.4\%) were of sub type $B$ (connection between FHL to 1st digital tendon of FDL; Fig: 2 ) and 2(11.8\%) were of sub type $\mathrm{C}$ (connection between FHL and 2 \&3rd digital tendons of FDL; Fig: 3).

Another noteworthy finding was that in one of the type 1 samples $1^{\text {stlumbrical received its }}$ origin from distal part of tendinous slip in addition to the medial side of $1^{\text {st }}$ digital slip of FDL (Fig: 2).

Fig. 1: Showing Type 5 pattern- Two tendinous slip from FHL to FDL.

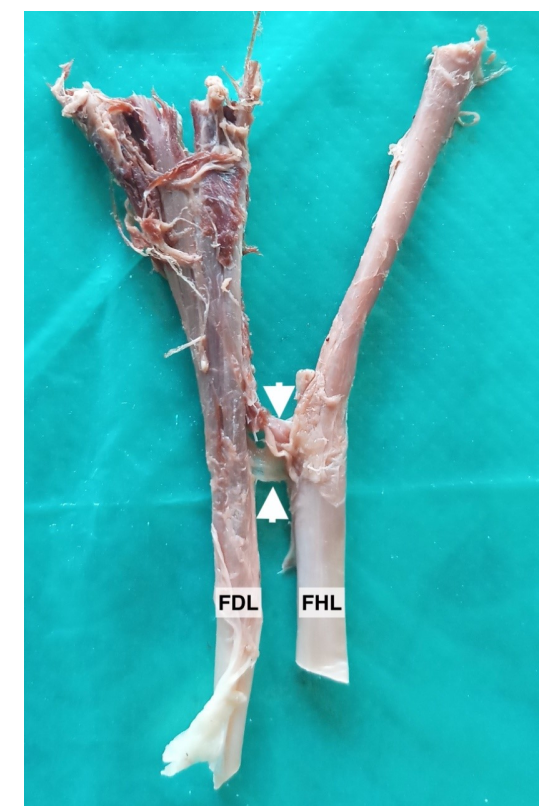

FHL: Tendon of Flexor hallucis longus, FDL: Tendon of Flexor digitorum longus, White arrow heads: Tendinous slips 
Fig. 2: Showing Type 1B pattern- tendinous slip from FHL to $1^{\text {st }}$ digital slip of FDL.

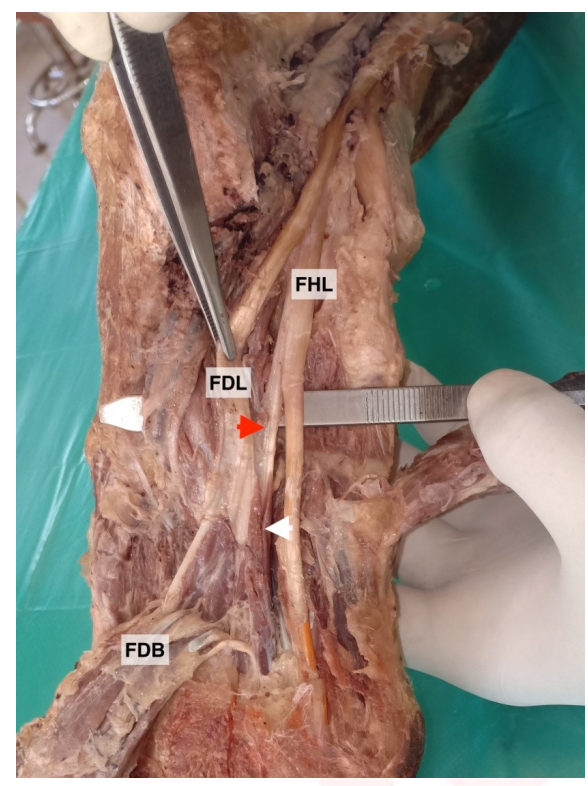

FHL: Tendon of Flexor hallucis longus, FDL: Tendon of Flexor digitorum longus, FDB: Flexor digitorum brevis, Red arrow head: Tendinous slip, White arrow head: $1^{\text {st }}$ Lumbrical sharing origin from tendinous slip

Fig. 3: Showing Type 1C pattern- Tendinous slip from FHL to $1^{\text {st }}$ and $2^{\text {nd }}$ digital slips FDL.

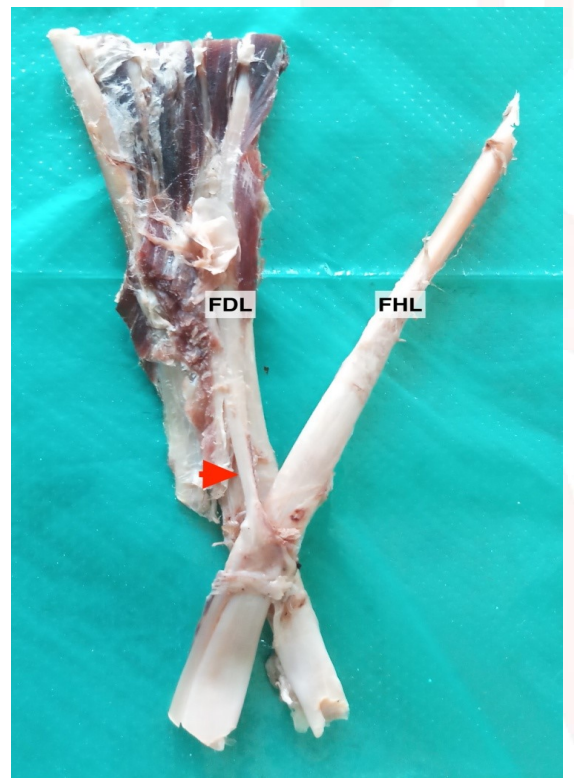

FHL: Tendon of Flexor hallucis longus, FDL: Tendon of Flexor digitorum longus, Red arrow head: Tendinous slip

\section{DISCUSSION}

The FHL and FDL are major tendons of the foot which originate from the common tendinous plate that may result in existence of various types of connections between them $[1,9]$. Moreover, their routine use in reconstructive surgeries of foot and ankle calls for compre hensive knowledge of their connections. There is a relative paucity of literature especially among Indian population. In view of the above this study was aimed to shed a fresh light on various types of connections between FHL and FDL.Various types of classifications were reported in the previous literature. Classification given by Orhan Beger et al [8] encompasses a wide range of connections. Therefore, this study used above classification to fit various types of connections between FHL and FDL. Present study has found only 3 types of connections among original 7 types described by Orhan Beger et al [8]. Of the 34 samples analysed, all of them had only 3 types of connections i.e. type 1 , type 4 and type 5 .

This study has found 17 type 1 (Connection from FHL to FDL) which accounts for the $50 \%$ of sample. There was only one study by LaRue BG et al [7], which was closer to the present findings with $41.6 \%$ of type 1 . All other studies had reported higher percentage of type 1 ranging from $67 \%$ to $97 \%[5,6,8,10,11]$. Studies with higher percentage of type 1 were carried out in higher sample size whereas LaRue BG et al [7] study had smaller sample size similar to present study. Interestingly, a linear relation between sample size and type 1 percentage was noted with studies with higher sample size had higher percentage of type 1 .

Type 4 (no connection between FHL \& FDL) was found in 16 specimens that accounted for $47.1 \%$. Most standard text books of anatomy state type 4 as normal pattern. Although considered as normal pattern it is interesting to note that most of the previous studies have not reported type 4 even with higher sample size $[5,6,8]$. There was only one study by LaRue BG et al [7] reported type 4 to be $16.6 \%$.

Only 1 sample was found to be Type 5 (double connections between FHL \& FDL) which was $2.9 \%$. Only a couple of studies had reported type 5 which was found to be $5 \%$ by Orhan Beger et al [8] and $11 \%$ by Mutsuaki Edama et al [6]. Type 1 was further divided in to $A, B$ and $C$ sub types. Sub type $1 \mathrm{~A}$ was a single tendinous connection proximally from FHL distally to the main stump of FDL which was accounted to be 10 (58.8\%) out 17 type1. Sub type 1B (connection between FHL to $1^{\text {st }}$ digital tendon of FDL) was found to be 5 (29.4\%) which was equivalent of sub type $1 \mathrm{~A}$ that was reported to be $45 \%$ by Christian Plaass et al [5] and $44 \%$ by 
Table 1: Comparison of distribution of various types of connections between FHL and FDL.

\begin{tabular}{|c|c|c|c|c|c|c|c|}
\hline \multirow{2}{*}{ Author } & Sample & \multicolumn{7}{|c|}{ Type of connection } \\
\cline { 5 - 9 } & size (n) & $\mathbf{1}(\%)$ & $\mathbf{4}(\%)$ & $\mathbf{5}(\%)$ & $\mathbf{1 A}(\%)$ & 1B (\%) & 1C (\%) \\
\hline Present study & 34 & 50 & 47.1 & 2.9 & 58.8 & 29.4 & 11.8 \\
\hline Orhan Beger et al [8] & 20 & 75 & 0 & 5 & -- & -- & -- \\
\hline Christian Plaass et al [5] & 60 & 67 & 0 & 0 & -- & 45 & 45 \\
\hline LaRue BG et al [7] & 24 & 41.6 & 16.6 & -- & -- & -- & -- \\
\hline Mutsuaki Edama et al [6] & 100 & 86 & 0 & 11 & & 44 & 62.7 \\
\hline Mao H et al [10] & 64 & 96.9 & -- & -- & -- & -- & -- \\
\hline Haijiao Mao et al [11] & 68 & 97 & -- & -- & -- & -- & -- \\
\hline
\end{tabular}

Mutsuaki Edama et al [6]. Sub type 1C (connection between $\mathrm{FHL}$ to $1^{\text {st }}$ and $2^{\text {nd }}$ digital tendons of FDL)was found to be in 2 samples (11.8\%) that was similar to sub type $1 \mathrm{~B}$ of Christian Plaass et al [5] and Mutsuaki Edama et al [6] classification that accounted for $45 \%$ and $62.7 \%$ respectively. Sub typing of type1 was not mentioned in majority of the previous literature except by Orhan Beger et al [8], Christian Plaass et al [5] and Mutsuaki Edama et al [6] to the best our knowledge. Orhan Beger et al [8] sub typing of type 1 was based on the contribution of FHL slips to long flexor tendons of the toes which was rather more of functional classification. Whereas the present study sub typed the type 1 based on anatomical connection which was duly noted and photographed.

It has been observed that in one of the type $1 \mathrm{~A}$ samples $1^{\text {st }}$ lumbrical received its origin from distal part of tendinous slip in addition to the medial side of $1^{\text {st }}$ digital slip of FDL. This is a novel finding of this study to the best of our knowledge. During reconstruction surgery FHL is freed from its connections which may lead to functional loss of digits. Our finding of lumbrical receiving dual origin poses a greater degree of functional loss during the surgery. Therefore, surgeon needs to pay more attention to lumbrical origin in view of this new finding. Disconnection of lumbrical leads to loss of gripping which play a major role in high intensity work outs of athletes.

\section{CONCLUSION}

This study provides a new insight into the types of connections between FHL and FDL. Only type1, 4 \& 5 were observed in the study with maximum distribution of type1 followed by type 4 (17 \& 16 out of 34 respectively). Among type 1 sub types type $1 \mathrm{~A}$ were more frequent followed by type $1 \mathrm{~B}$ and $1 \mathrm{C}$. This study also reports for the first time a common origin of $1^{\text {st }}$ lumbrical from distal part of tendinous slip as well as from $1^{\text {st }}$ digital slip of FDL. These observations warrant further studies with large sample to substantiate the findings of our study and to know the degree of distribution especially in multi ethnic society such as India.

\section{Conflicts of Interests: None}

\section{REFERENCES}

[1]. Parul Kaushal, AritraBanerjee, PushpaDhar. Knot of Henry: Role in Tendon Transfer. Journal of Clinical and Diagnostic Research. 2017;11(12): 01-02.

[2]. Julien Wegrzyn, Jean-François Luciani, Rémi Philippot, Elisabeth Brunet-Guedj, Bernard Moyen $\&$ Jean-Luc Besse. Chronic Achilles tendon rupture reconstruction using a modified flexor hallucis longus transfer. International Orthopaedics (SICOT) 2010; 34:1187-1192.

[3]. Amlang M, Rosenow MC, Friedrich A, Zwipp $H$, Rammelt S. Direct plantar approach to Henry's Knot for flexor hallucis ongus transfer. Foot Ankle Int. 2012;33(1):7-13.

[4]. DiPaola M, Raikin SM. Tendon transfers and realignment osteotomies for treatment of stage II posterior tibial tendon dysfunction. Foot Ankle Clin. 2007;12(2):273-285.

[5]. Christian Plaass, Ghassan Abuharbid, Hazibullah Waizy, Matthias Ochs, Christina StukenborgColsman, and Andreas Schmiedl. Anatomical Variations of the Flexor Hallucis Longus and Flexor Digitorum Longus in the Chiasma Plantare. Foot \& Ankle International 2016;34(11):1580- 1587.

[6]. Mutsuaki Edama, Masayoshi Kubo, Hideaki Onishi, Tomoya Takabayashi, Erika Yokoyama, Takuma Inai, Hiroshi Watanabe, Satoshi Nashimoto, Ikuo Kageyama. Anatomical study of toe flexion by flexor hallucis longus. Annals of Anatomy 2016;204:8085.

[7]. LaRue BG, Anctil EP. Distal anatomical relationship of the flexor hallucis longus and flexor digitorum longus tendons. Foot Ankle Int. 2006;27:528-32. 
[8]. Orhan Beger, Ozlem Elvan, Mert Keskinbora, Burçin Ün, Deniz Uzmansel, Zeliha Kurtoglu. Anatomy of Master Knot of Henry: A morphometric study on cadavers. Acta Orthopaedica et Traumatologica Turcica 2018;52:134e142.

[9]. Yamamoto $M$, Shiraishi $Y$, Kitamura K, Jin ZW, Murakami G, Rodríguez-Vázquez JF, Abe SI. Early embryonic development of long tendons in the human foot. Okajimas Folia Anat Jpn. 2016;93(2):5965.
[10]. Mao H, Shi Z, Wapner KL, Dong W, Yin W, Xu D. Anatomical study for flexor hallucis longus tendon transfer in treatment of Achilles tendinopathy. Surg Radiol Anat. 2015;37(6):639-647.

[11]. Mao H, Dong W, Shi Z, Yin W, Xu D, Wapner KL. Anatomical Study of the Neurovascular in Flexor Hallucis Longus Tendon Transfers. Scientific reports. 2017 Oct 27;7(1):14202.

How to cite this article:

V. Manikanta Reddy, V. Vallishri, C. K. Lakshmi Devi. STUDY ON TENDINOUS CONNECTIONS BETWEEN FLEXOR HALLUCIS LONGUS AND FLEXOR DIGITORUM LONGUS. Int J Anat Res 2019;7(2.2):6531-6535. DOI: 10.16965/ijar.2019.161 